\title{
EXAMINING THE EFFECTIVENESS OF VIRTUAL COMMUNITIES OF PRACTICE: A RESEARCH FRAMEWORK
}

\author{
Dr. Sean T. McGann - Ohio University MIS Department - mcgann@ohio.edu \\ Dr. Hala Annabi - Ohio University MIS Department - annabi@ohio.edu
}

\begin{abstract}
The paper suggests a research framework focused on assessing the viability of virtual communities of practice (VCOP) as an effective means of knowledge management. While the dearth of current research on VCOP and knowledge management has suggested a need for personal contact in order to facilitate effective knowledge transfer, research in this area is limited. It is our contention that certain types of communities of practice can be successful without face-to-face contact, but additional research is needed to substantiate this claim. To promote this stream of research, the key foundational concepts of VCOP: communities of practice, virtual teams and knowledge management are reviewed and mapped to a framework designed to drive future research in the area of VCOP.
\end{abstract}

Keywords: Virtual communities of practice, Virtual teams, Knowledge management, Virtual learning

\section{INTRODUCTION}

Increased emphasis on knowledge as an imperative to organizational effectiveness, the growth of communities of practice (COP) as a key structure to facilitate knowledge transfer and the advent of the Internet as a powerful collaborative learning tool has given rise to research on virtual communities of practice (VCOP), which combines all of the above areas (Cashel 2001). It is no mystery that an important transformation has taken place in the past 10 years, which has shifted the focus from 'information' to 'knowledge' as the key to organizational effectiveness. The translation of unique experiences into knowledge is what assures organizations will be able to repeat past successes, avoid future failures, and evolve in the process (Dixon 2000). As a result, a viable knowledge management strategy and the means to implement it has become an organizational imperative.
A key issue that follows is how to effectively capture and leverage all knowledge, but especially tacit knowledge. This is where the notion of organizational structuring comes into play. Lave and Wenger's 'community of practice', introduced in 1991, has received much recognition in recent organizational literature. These communities have been shown to be extremely effective in the promotion of tacit knowledge. In his assertion about the importance of communities of practice, Wenger stresses that "information stored in explicit ways is only a small part of the picture and that true knowing and learning is something that comes about by participation in communities". He further states that communities of practice are an 'intrinsic condition for the existence of knowledge' (Lave and Wenger 1991).

Having established the importance of knowledge management, and leveraging COPs to provide the organizational backdrop, the core issue that remains is how to design and implement a COP for explicit and especially tacit knowledge management in modern organizations. As organizations have become more geographically disbursed due to globalization, mergers and acquisitions, and general growth strategies, the use of virtual collaboration to share knowledge is becoming an imperative (Tapscott 2006). It is certainly the case today that we live in a world which is increasingly embracing the use of information technology to bolster its collaborative capabilities. The use of information technology to leverage virtual communication channels for knowledge offers the benefits of decreasing cost (e.g. decreased travel/logistics) and increasing efficiency (e.g. increased access/ability to contribute) of the knowledge management process. Further, as more organizations are adopting COP as a means of transferring knowledge, more frequent collaboration and increased flexibility is necessary. Therefore, it does not seem difficult to build an effective case for the need for VCOP. 
The key point of controversy and the basis for this research proposal is the question of whether communities of practice can effectively exist in a purely virtual situation. Therefore, a research stream targeted at the examination of VCOPs, their different types and characteristics, what drives them to success, what their limitations are, and what the future holds for them is proposed. The proposed research stream seeks to answer a host of related research questions through our research framework which is developed below.

This paper contributes to existing research in the area of VCOPs in a number of important ways. First of all, it serves as an extension of Kimble et al's (1998, 2000, 2001) work, focusing on VCOPs, which rely on face-to-face contact. It is also expected to uncover additional data, which would be helpful in expanding the knowledge base for COPs in general. The research is grounded largely in the virtual teams research of Goddard (1992), Hepworth (1989) and $\mathrm{Li}$ (1995). As such, it should provide a fresh perspective on virtual teams, with the integration of the COP. It also seeks to move towards building a methodology for practicioners to leverage in the design and implementation process for VCOPs.

\section{LITERATURE REVIEW}

\section{Communities of Practice}

Community of practice research has been pioneered by Lave and Wenger and was first introduced in 1991. The definition offered by this team was as follows: "a set of relations among persons, activity, and the world over time and in relations with other tangential and overlapping communities of practice”. In such a community, participation in activities through a master/student relationship is key to the social learning process. This participation takes place within an activity that all members have a common understand of. In this process, notions such as belonging through engagement in it, imagination or constructing an image of the community to orient members, and alignment of activities with the goals of the community, provide the foundation. In examining the dimensions of COPs, Lave and Wenger also emphasizes enterprise (level of learning energy), mutuality (depth of social capital), and repertoire (degree of self-awareness) as keys to a strong community. The notion of learning at the 'fluid' boundaries of COPs is also incorporated into Wenger's work in an effort to show the opportunities created when experience of members and their competence come into close tension. (Wenger 1998) The last component that Wenger emphasizes is the identity of the community and its crucial nature in the social learning process, emphasizing that without it, knowing and learning does not take place.

Since then, other definitions of COP have surfaced, which seem to be a result of the dynamic business environment of late. One such definition was offered by Manville and Foote (1996):

"a group of professionals informally bound to one another through exposure to a common class of problems, common pursuit of solutions and thereby themselves embodying a store of knowledge”

These and other interpretations are still built upon the same fundamental concepts of Wenger, but applied to a wide array of business groups such as departments, functional teams, and project teams (Sandusky 1997).

\section{Implications for our VCOP Research Framework}

It is our contention that the concepts embodied in the study of communities of practice seem to be general enough to map to VCoPs. Questions regarding the level of 'belonging' to VCoPs, the 'dimensions' of VCoPs, the nature of the 'boundaries' of VCoPs and finally their ability to establish a sufficient 'identity' to exist on their own are central.

\section{Virtual Teams}

An aggregate definition of virtual teams specifies them as a discrete geographically disbursed group of workers or members brought together to accomplish a specific organizational task using information technology as the medium of interaction. The team members can be separated by physical and/or temporal borders and can be from different organizations (Townsend et al 1998; Markus et al. 2000, Bélanger et al.1998).

In understanding virtual teams, two key issues that were pervasive in the literature were the need to assess trust and identity (Kimble et al, 2000; Grawbowski and Roberts 1998, Handy 1995). The assertion was made poignantly that without trust in a "faceless environment", virtual teams will not succeed (Handy 1995). Further, in 
an environment where information is present instead of matter, the establishment of an identity is a great challenge. Many of the basic cues about personality and trustworthiness are absent (Kimble et al, 2002).

\section{Areas for our VCOP Research Framework}

The primary questions with regards to VCOP that this research brings to light are along these lines: Can trust be established in a VCOP? Or, can a VCOP that requires a great amount of trust (e.g. one that involves the exchange of sensitive information) survive? Can an acceptable level of identity be established in a VCOP?

\section{Knowledge Management}

The primary focus from a knowledge perspective with regards to VCoPs is whether they can be effective for the preservation and transfer of explicit AND tacit knowledge. Much of the knowledge management (KM) literature is focused on explicit, or 'know what' knowledge which is easily captured-codified and stored. As such, it lends itself well to the use of information technology to be managed (Kimball et al, 2000). This fact helps to support the argument for VCOPs, until one considers that this type of knowledge is less vital in promoting organizational effectiveness, as it lacks the vital experiential component that is embedded in the practices (Lave and Wenger 1991). Further in a competitive environment, it is the type of knowledge that is largely available across organizations and thus does not provide a distinct competitive advantage (Dixon 2000).

However, the 'know how', or tacit knowledge, is the precious commodity that is getting more recognition in KM literature. It is touted as the more vital component of knowledge (Wenger 1998), as it allows organizations to make the transition from simply having step-by-step instructions, by introducing realities such as cultural, experiential and environmental issues that are often not easily articulated. Therefore, it is said to promote an understanding of the deeper issues of organizational situations and processes. An additional viewpoint offered in the literature explains the source of legitimacy of knowledge as an important distinguishing factor. While explicit knowledge is accepted as legitimate by virtue of formal authority and is thus less prone to true acceptance, tacit knowledge gains acceptance by informal authority or consensus of the community, and is thus the truly accepted source of meaning. (Kimball et al 2000) Further review showed that tacit knowledge transfer is largely seen to be an event that only takes place in a face-to-face context. (Kimball et al 2000)

\section{Areas for our VCOP Research Framework}

Tacit knowledge seems to be the more valuable commodity in the KM realm, but also the more difficult to capture and preserve, even in a nonvirtual context. The question then becomes, with regards to VCOPs: Can VCOPs effectively capture and transfer tacit knowledge? The assumption for purposes of this research, which is supported by the literature is that VCOPs CAN effectively capture and transfer explicit knowledge.

\section{Virtual Communities of Practice}

Our review showed that research on VCOPs seems to be in the early stages. As such, we found their conclusions were not supportive of strong generalizations. Studies that were reviewed were conducted on limited sample size of organizations, in limited contexts and across short time frames. Most of the work to date specifically on VCOPs has been case study based and conducted by different combinations of the team of Kimble, Hildreth, Wright, Li and Barlow (1999, 2000, 2001) on large commercial organizations with distributed work teams. In reality, their studies were not on "pure" VCOPs as defined by this paper, but on distributed work teams that used a combination of face-to-face and information technology as a mode of operation. Their limited generalizations support the importance of tacit knowledge in VCOPs, but indicate that face-to-face contact is a necessity in order for virtual teams to succeed.

From a practical perspective, an interview with Richard McDermott (Cashel 2001), a renowned COP practicioner, cited claims that virtual communities of practice could potentially "make information and knowledge free commodities, democratize the workplace, create relationships and remove barriers of status" as hype. His conclusion was that the Internet is just one means of connecting in VCoPs, and that good communities use all means available for the kind of information and experience that they want to share (such as face to face, fax, email, etc). Again, the assertion is made that face-to-face contact cannot be replaced by information technology. 
And finally from an Ecommerce-based perspective, Durlacher.com, an online community research site, provides an interesting definition: VCOPs are communities of people who share the same profession, situation, or vocation. These communities facilitate professional exchange, allow members to establish a bond of common experiences and challenges, and build networks of relationships, which may be leveraged offline. The community of parents on ParentSoup, Medical Professionals on Healtheon/WebMD or Java programmers on EarthWeb are examples of VCoP. They go on to point out that VCOP are particularly lucrative in the business-to-business space as they offer considerable opportunities for the translation of community into commerce revenues. In their articles they argue that VCOPs are not simply a 'nice to have' adjunct for a web-based business, but that their creation is central to a sustainable business model. They conclude by expanding on some of the key success factors for a VCOP in this realm as: self-generated evolution, involvement and interactivity, frequency and duration of visits. (Durlacher 1999) From this perspective, there is much less emphasis on faceto-face interaction as a success factor and more on leveraging information technology to create interaction and opportunities for Ecommerce.

\section{Areas for our VCOP Research Framework}

As the above review shows, the VCOP research stream is still in its early stages and thus represents a great opportunity for future research. The primary questions that seem to stem from this research are: Is face-to-face contact is necessary to establish effective COPs? In which community contexts/themes (professional, personal, etc) is face-to-face contact more of a factor?

\section{OUR VCOP RESEARCH FRAMEWORK}

Through the previous sections of this paper, it was our intent to show the vital nature of knowledge as driver for organizational effectiveness, COPs as an organizational structure that promotes knowledge creation and transfer, and the potential opportunities that information technology provides through the use of VCOPs. In the next section the research questions from the literature review are broken down as follows in Table 1 into a set of dependent and independent variables to drive future research. We see these as potential measures of VCOP success through increased levels of the dependent variables below:

\begin{tabular}{|l|l|}
\hline Independent Variables & Dependent Variables \\
\hline Professsional vs. personal interest themes & Amount of learning that takes place \\
\hline Partially vs. totally virtual & Level of participation \\
\hline Amount of face-to-face contact & Level of tacit knowledge transfer \\
\hline Level of formality & Level of established trust \\
\hline Virtual from inception vs. transfer to virtual & Depth of overall identity \\
\hline Exclusivity of membership & Sense of belonging \\
\hline Sensitivity of information exchanged & \\
\hline Level of technical expertise of members & \\
\hline
\end{tabular}

\section{Table 1 - Research Variables}

The next step was to map these variables to devise a framework of the key concepts entailed in knowledge management, COPs, virtual teams, which outlines the key issues that lie at the heart of the study of VCOPs. We then use the framework to drive key research questions and hypotheses in the following section. The framework in Table 2 is a compilation of all the VCOP foundational concepts that were reviewed along with their key issues. We also created a more detailed list of potential research questions, which were based on the framework. 


\begin{tabular}{|c|c|c|}
\hline $\begin{array}{l}\text { Foundational } \\
\text { Concept }\end{array}$ & Inherent Issues & Research Questions for Further Study \\
\hline $\begin{array}{l}\text { Communities of } \\
\text { Practice }\end{array}$ & $\begin{array}{ll}\text { - } & \text { Belonging } \\
\text { - } & \text { Dimensions } \\
\text { - } & \text { Boundaries } \\
& \text { Identity }\end{array}$ & $\begin{array}{l}\text { - Can VCOPs provide an environment that will promote } \\
\text { establishment of strong engagement, imagination and } \\
\text { alignment? If so, what are the characteristics of such a } \\
\text { community? } \\
\text { - Is there sufficient learning energy, social capital and } \\
\text { repertoire developed in a VCOP setting? } \\
\text { Are there sufficient boundaries, which allow tension } \\
\text { between experience and competence to provide learning } \\
\text { opportunities in VCOPs? } \\
\text { Can a group in a VCOP setting establish as strong } \\
\text { identity? }\end{array}$ \\
\hline $\begin{array}{l}\text { Knowledge } \\
\text { Mangement }\end{array}$ & $\begin{array}{ll}\text { Effective tacit } \\
\text { knowledge transfer }\end{array}$ & $\begin{array}{l}\text { - What are the conditions necessary to allow for tacit } \\
\text { knowledge transfer in VCOPs? } \\
\text { - How can technology be leveraged most effectively in } \\
\text { VCOPs to facilitate tacit knowledge transfer? }\end{array}$ \\
\hline Virtual Teams & $\begin{array}{ll}\text { - } & \text { Trust } \\
\text { - } & \text { Identity }\end{array}$ & $\begin{array}{l}\text { - Do VCOPs provide an environment more conducive to } \\
\text { trust than virtual teams? } \\
\text { Does the lack of a strong identity create lack of trust in } \\
\text { VCOPs? }\end{array}$ \\
\hline $\begin{array}{l}\text { Virtual } \\
\text { Communities of } \\
\text { Practice }\end{array}$ & $\begin{array}{ll}\text { - } & \text { Face-to-face contact } \\
\text { - } & \text { Community Themes }\end{array}$ & $\begin{array}{l}\text { Can VCOPs be effective without face-to-face contact? } \\
\text { Is a VCOP that has been virtual since its inception more } \\
\text { likely to succeed than one that has made a transition to } \\
\text { virutality? } \\
\text { - Which community themes lend themselves to VCOP } \\
\text { structure? (e.g. personal vs. professional) } \\
\text { How does the level of tech experience influence the } \\
\text { success of VCOPs? }\end{array}$ \\
\hline
\end{tabular}

Table 2 - VCOP Framework

\section{PROPOSITIONS}

Driven by the compilation of concepts in the previous section, we now propose three hypotheses for further research. Although this is obviously not an exhaustive list based on the framework, we deemed those selected as most testable and interesting based on our research and past experience.

\section{Proposition 1}

VCOPs that originate as solely virtual will be successful more often than those that transition to a virtual mode of operation.

It is our contention that VCOPs designed without face-to-face contact in mind will be more likely to have the processes, technologies and expectations set in a fashion that will assure their success more readily than a COP trying to transition to a virtual model. This could be tested using a longitudinal case study of a number of VCOPs that have been solely virtual since inception and comparing their successes to those that have transitioned.

\section{Proposition 2}

VCOPs that involve themes of personal interest will be successful more often than those that are organized around a professional work team. Having participated in both types of VCOPs, and experienced the issues involved, it is our contention that the energy, enthusiasm and inherent trust that is present in a VCOP whose theme is based on personal passion, is more often stronger than one of a purely professional nature. Often in the personal case involving a deep area of interest, a bonding phenomenon takes place solely by virtue of the fact that a deep passion is shared. This facilitates trust, identity, and host of other favorable aspects cited in the literature as only evolving through face-to-face contact. In this case, the ability to connect with a large community that shares this passion compensates for the lack personal contact. This condition could be tested by comparing the 
above success variables of a number of established VCOPs that have personal themes with those that have professional themes.

\section{Proposition 3}

VCOPs will be successful more often than virtual teams in accomplishing the same goal.

It seems that the problems arising in virtual teams are largely resolved by the common ground that is provided by VCOPs. Trust and identity problems are less prevalent and will therefore assure success more often in VCOPs than in virtual teams. This hypothesis could be tested similarly to the previous condition by measuring the success of a number of VCOPs, with similarly tasked virtual teams.

\section{CONCLUSION}

In reviewing the current state of affairs for VCOP, as well as predicting their future, it seems that they are largely misunderstood and underestimated. Although the research conducted to date has not shed favorable light on pure VCOPs, it is again important to reiterate that this research is limited. As such, this area represents a significant opportunity for researchers and practicioners alike. As the use of the Internet as a collaborative medium increases, and organizations learn to embrace the power of VCOPs as a structure for knowledge management, it is our contention that a culture will evolve which is less dependent on face-toface contact and more adept at leveraging purely information technology driven forums like VCOPs to accomplish organizational goals.

\section{REFERENCES}

Cashel, J. (2001). Interview with Richard McDermott, Forum One Communications. February.

Dixon, N. (2000). Common Knowledge. Boston: Harvard School Press.

Durlacher, J. (1999). Creating Community Online. Durlacher.com. February.

Bélanger, F. and Collins, R. (1998). Distributed Work Arrangements: A Research Framework. The Information Society, 14, 137-152.

Goddard, J. (1992). New technology and the geography of the UK information economy. In Robins, K. (ed) Understanding information business technology and geography. Belhaven London.

Grabowski, M. and Roberts, K. H. (1998). Risk mitigation in virtual organizations. Journal of Computer Mediated Communication, 3. Available: http://jcmc.huji.ac.il/vol3/issue4/grabowski. html.

Handy, C. (1995). Trust and the virtual organization. Harvard Business Review, 73(3): 40-50.

Hepworth, M. (1989). Geography of the information economy. Belhaven: London.

Hildreth, P., Kimble, C., Wright, P.(1999). Knowledge Management: Are we Missing Something? Information Systems: The Next Generation, UKAIS Proceedings, 347-356, April.

Kimble, C., Li, F. and Barlow, A. (2000) Effective virtual teams through communities of practice. Glasgow: University of Strathclyde, Strathclyde Business School. (Management Science Research Paper No. 00/9). Available: http://ssrn.com/abstract=634645

Kimble, C., Hildreth, P. and Wright, P. (2001). Communities of practice: going virtual. In Malhotra, Y. (ed.) Knowledge management and business innovation. Hershey, PA: Idea Group. Available: http://wwwusers.cs.york.ac.uk/ kimble/research/13kim ble.pdf

Lave, J. and Wenger, E. (1991). Situated learning. Legitimate peripheral participation. Cambridge: Cambridge University Press.

LI, F. (1995). The Geography of Business Information, John Wiley and Son, Chichester.L. and Kimble C. (Eds.) Information systems - the next generation. Proceedings of the fourth UKAIS conference. York UK. pp471-487.

Markus, M. L., Manville, B. and Agres, E. C. (2000) What makes a virtual organization work? Sloan Management Review, 42, 1326.

Malhotra, A., Gosain, S. and Hars, A. (2001) Evolution of Virtual Community: Understanding Design Issues Through a Longitudinal Study. ECIS Proceedings. 
Manville, B. and Foote, N. (1996). Harvest your workers' knowledge. Datamation. Available:

http://www.datamation.com/PlugIn/Issues/1 996/July/07know1.html.

Hildreth P, Kimble C and Wright P. (1998). Computer Mediated Communications and Communities of Practice. Proceedings of Ethicomp'98, Erasmus University, The Netherlands, pp 275 - 286.

Sandusky, R. J. (1997). Infrastructure Management as Cooperative Work. Proceedings of International ACM, pp 91100.

Tapscott, D. \& Williams, A. (2006). Wikinomics : How mass collaboration changes everything . New York: Portfolio.

Townsend, A.M., DeMarie, S.M., and Hendrickson, A.R. (1998). Virtual teams: Technology and the workplace of the future. Academy of Management Executive, 12(3), 17-29.

Wenger, E. (1998). Communities of Practice: Learning, Meaning, and Identity. Cambridge: University Press.

Wenger, E. (2000) Communities of Practice and Social Learning Systems. Organizational Articles, 7(2).

Wenger, E., McDermott, R., and Snyder, W. (2002). Cultivating Communities of Practice. Cambridge, MA: Harvard Business School Press. 